\title{
OPEN Influence of ultrasound on juvenile hormone titers in Monochamus alternatus Hope (Coleoptera: Cerambycidae)
}

\author{
Yu-Ping Zha ${ }^{1 \bowtie}$, Xiao-Ling Wu ${ }^{2}$, Zi-Yi Zhang ${ }^{1}$, Jing-Yuan Chen ${ }^{1}$ \& Qi-Cai Chen ${ }^{2}$
}

Abiotic stress factors can significantly affect insects. In particular, the stressful effects of exposure to ultrasound on insects are considered important. In the present study, we investigated the effects of ultrasound on the important global pest Monochamus alternatus (Coleoptera: Cerambycidae), which is the main vector of the pinewood nematode. We exposed $M$. alternatus adults (aged 1 day, 3 days, and 5 days) to ultrasound at different frequencies (using two ultrasonic devices, i.e., LHC20 with a mixture of frequencies at $35 \mathrm{kHz}, 70 \mathrm{kHz}$, and $105 \mathrm{kHz}$; and GFG-8016 at two separate frequencies of $30 \mathrm{kHz}$ and $60 \mathrm{kHz}$ ) for different periods of time $(1 \mathrm{~h}, 12 \mathrm{~h}$, and $24 \mathrm{~h})$, before evaluating the juvenile hormone III (JHIII) titers. All of the ultrasound treatments significantly decreased the JHIII titers in M. alternatus adults. The decreases in the JHIII titers due to ultrasound exposure did not differ according to sex, but the effects on beetles of different ages differed significantly depending on the duration of exposure. The decreases in the JHIII titers were highest in male and female beetles after exposure to ultrasound for $12 \mathrm{~h}$. Following exposure to ultrasound for any time period, the decreases in the JHIII titers were lower in adults aged 3 days than those aged 1 day and 5 days. The different ultrasonic frequencies led to variable decreases in the JHIII titers in $M$. alternatus adults, where the greatest decreases occurred in beetles exposed to ultrasound at $60 \mathrm{kHz}$. Our results indicate that ultrasound can negatively affect the normal JHIII levels and it may further disrupt sexual maturation by $M$. alternatus adults.

Juvenile hormone $(\mathrm{JH})$ is a vital hormone that regulates reproduction, development, and behavior in insects ${ }^{1-3}$. $\mathrm{JH}$ plays primary roles in reproductive processes by stimulating or inhibiting reproductive development, although the extent and timing of the actions of $\mathrm{JH}$ may vary among different insects ${ }^{4}$. There are over four types of $\mathrm{JH}$, where JHIII is the most common in Coleoptera ${ }^{5}$. In general, changes in the JH levels can regulate the development of insects, e.g., the JH levels are low at eclosion and higher in the adult of the mosquito Aedes aegypti (L.) (Diptera: Culicidae) ${ }^{2}$. In the locust Locusta migratoria migratorioides R. and F. (Orthoptera: Acrididae), JH has an important function in the longitudinal muscle developmental process at the time of oviposition in females ${ }^{6}$. $\mathrm{JH}$ is required for vitellogenin synthesis in the beetle Tribolium castaneum Herbst (Coleoptera: Tenebrionidae) and it remains at a high level 1-5 days after adult emergence ${ }^{7} . \mathrm{JH}$ is involved in mating behavior, aggregation behavior, and other behaviors in insects ${ }^{1,8-10}$. In particular, $\mathrm{JH}$ can affect the sensitivity of the olfactory antennal lobe neurons in males of the moth Agrotis ipsilon (Hufnagel) (Lepidoptera: Noctuidae) to modulate the timing of mate recognition ${ }^{11}$. Physiological evidence indicates that the $\mathrm{JH}$ may inhibit the activity of acetylcholinesterase in insect cells ${ }^{12}$.

Monochamus alternatus is the main vector of the pinewood nematode, Burasphelenchus xylophilus (Steiner and Buhrer) (Nematode: Aphelenchoididae), which causes serious damage to pines ${ }^{13}$. At present, there are no effective strategies for controlling M. alternatus, such as physical, chemical, or biological methods ${ }^{14}$. The application of acoustic technology in pest management is increasingly very rapidly based on techniques for producing signals that disrupt vibrational communication, as well as the development of control treatments by combining pheromones with precisely patterned sonic or vibrational signals ${ }^{15}$. Ultrasound $(35-105 \mathrm{kHz})$ can significantly affect insects, where it can reduce the amount of eggs laid and the mating time for adult Plodia interpunctella (Hübner) (Lepidoptera: Pyralidae), as well as modulating the acetylcholinesterase and antioxidant enzyme systems in Helicoverpa armigera (Hübner) (Lepidoptera: Noctuidae $)^{16-18}$. The ultrasound $(60-80 \mathrm{kHz})$ also had a

${ }^{1}$ Hubei Academy of Forestry, Wuhan 430075, People's Republic of China. ${ }^{2}$ College of Life Sciences and Hubei Key Lab of Genetic Regulation and Integrative Biology, Central China Normal University, Wuhan 430079, People's Republic of China. ${ }^{凶}$ email: zhayuping@163.com 
significant influence on the flight behavior of Myrmeleon hyalinus (Neuroptera Myrmeleontidae) ${ }^{19}$. M. alternatus is a typical nocturnal animal ${ }^{20}$ and it may be affected by the ultrasound produced by Eptesicus serotinus (Chiroptera: Vespertilionidae), which is a widespread insectivorous bat in China that mainly feeds on beetles ${ }^{21}$.

In our previous study, we found that ultrasound affected the acetylcholinesterase activity in M. alternatus ${ }^{22}$ and JH may play an important role in this process. To test this hypothesis, in the present study, we investigated whether exposure to ultrasound at different frequencies and times might affect the JHIII titers in M. alternatus adults.

\section{Materials and methods}

Insects. Newly infested Pinus massoniana bolts were collected from a forest in Songzi, Hubei, China $\left(30^{\circ} 02^{\prime}\right.$ $43^{\prime \prime} \mathrm{N}, 111^{\circ} 45^{\prime} 29^{\prime \prime} \mathrm{E} ; 64 \mathrm{~m}$ above sea level). The bolts were transported to the forest entomology laboratory at Hubei Forestry Academy, Wuhan, China. The bolts were kept in a climate chamber at a temperature of $25-28^{\circ} \mathrm{C}$, relative humidity $(\mathrm{RH})$ of $45-55 \%$, and with a light:dark photoperiod of 16:8 h. Between early May and the end of June, the adult beetles that emerged from the bolts were collected each day and maintained separately in a cylindrical insect jar (diameter $=5.8 \mathrm{~cm}$, height $=8.7 \mathrm{~cm}$; Guangying Technology Co. Ltd, China). Fresh P. massoniana twigs aged one year were provided to feed the beetles every day.

Ultrasonic devices. Two ultrasonic devices were evaluated comprising LHC20, a commercial device manufactured by Lihui, Inc., Wuhan, China ${ }^{17}$, and a function generator (GFG-8016G; Good Will Inst. Co. Ltd, Bayan Lepas, Penang, Malaysia). The LHC20 system could only generate one type of ultrasound with a mixture of peak frequencies at $35 \mathrm{kHz}, 70 \mathrm{kHz}$, and $105 \mathrm{kHz}$. The GFG-8016G instrument could generate ultrasound at two frequencies of $30 \mathrm{kHz}$ and $60 \mathrm{kHz}$. All of these frequencies are in the range for E. serotinus ${ }^{23}$. Ultrasound properties were measured using an ultrasound detector (D.1000X; Pettersson Elektronik AR, Sweden). Data were captured using a laptop computer equipped with a DAQCARD-AI-16E-4 acquisition card (National Instruments, Austin, Texas, USA). Data were collected at a rate of 200,000 samples/s and with 12-bit resolution. Sound pressure level (SPL) measurements were acquired based on 10 readings at any given position. SPL measurements were expressed in decibels with respect to 20 micropascals $(\mu \mathrm{P} ; 0 \mathrm{~dB}=20 \mu \mathrm{P}$ ).

All of the sound parameters generated by the function generator were fully programmable via a computer. The mean SPL \pm standard error (SE) produced was $94.6 \pm 0.3 \mathrm{~dB}$ at a distance of $50 \mathrm{~cm}$, and the ultrasound pulse width was $500 \mathrm{~s}$.

Test procedures. Monochamus alternatus adults were placed individually in $100 \mathrm{~mL}$ plastic containers (height $=12 \mathrm{~cm}$, diameter $=8 \mathrm{~cm}$ ). The open ends of the containers were tightly covered with wire mesh screens (opening area $=30 \mathrm{~cm}^{2}$ ). The LHC20 system or the function generator were placed $50 \mathrm{~cm}$ above the wire mesh covering the containers. The SPLs immediately above the wire mesh were $92.1 \pm 0.4 \mathrm{~dB}$ for the LHC20 device and $94.6 \pm 0.3 \mathrm{~dB}$ for the function generator. The control treatments comprised separate containers with $M$. alternatus adults, which were not exposed to ultrasound.

1. Healthy beetles (aged 1-13 days) were divided into 26 groups with three adults in each group according to their sex and age. All beetles were used for extracting JHIII.

2. To test the chronic effects of ultrasound, healthy beetles were divided into 18 groups with three adults in each group according to their sex and ages (1 day, 3 days, and 5 days), and the groups were then exposed individually to ultrasound produced from the LHC20 device for periods of $1 \mathrm{~h}, 12 \mathrm{~h}$, or $24 \mathrm{~h}$. Each treatment was replicated three times. Each test group had a corresponding individual control group. All tests were conducted at $25 \pm 1{ }^{\circ} \mathrm{C}, \mathrm{RH} 65 \% \pm 5 \%$, and under a light:dark photoperiod of $12: 12 \mathrm{~h}$.

3. To test the effects of different ultrasound frequencies, healthy beetles (aged 1 day) were divided into six groups with three adults in each group according to their sex. Individuals in these groups were then exposed to ultrasound produced by the LHC20 device or function generator for $12 \mathrm{~h}$. All tests were conducted at $25 \pm 1{ }^{\circ} \mathrm{C}$, RH $65 \% \pm 5 \%$ and with a photoperiod of $12: 12 \mathrm{~h}$.

Extraction and quantification of JHIII. After each treatment, individuals were cleaned with distilled water and dried using pieces of filter paper. Each sample insect was then weighed. JHIII was extracted from each sample according to the method described by Jiang ${ }^{24}$ with a few modifications. Each whole adult was mixed with $4 \mathrm{~mL}$ of methanol:ethyl ether $(1: 1, \mathrm{~V} / \mathrm{V})$ and homogenized in a micro-mortar using a micro-glass tube for $5 \mathrm{~min}$. The mixture was transferred to $10 \mathrm{~mL}$ centrifugal tubes and sonicated for $5 \mathrm{~min}$ with a UP200S ultrasonic processor (Hielscher, Germany), before adding $2 \mathrm{~mL}$ of $\mathrm{n}$-hexane, sonicating again for $5 \mathrm{~min}$, and centrifuging at $4000 \mathrm{r} / \mathrm{min}$ and $4{ }^{\circ} \mathrm{C}$ for $10 \mathrm{~min}$. The upper $\mathrm{n}$-hexane phase was collected and the lower layer was subjected to ultrasound-assisted re-extraction five times where $2 \mathrm{~mL}$ of $\mathrm{n}$-hexane was added after each sonication treatment. At the end of each centrifugation step, the $n$-hexane phases were mixed together. The combined $n$-hexane extracts were dried under nitrogen and dissolved in $500 \mu \mathrm{L}$ methanol, before filtering through a $0.22 \mu \mathrm{m}$ Millipore Express PES Membrane, and storing at $-20{ }^{\circ} \mathrm{C}$ until their analysis.

The JHIII titers were determined by high-performance liquid chromatography (HPLC) using a DGU-20A5 (Japan) system equipped with a Luna $5 \mu \mathrm{m} \mathrm{C18} \mathrm{column}(250 \mathrm{~mm} \times 4.6 \mathrm{~mm})$ and a flow-through ultraviolet (UV) detector. JHIII was eluted with $80 \%$ methanol in water at a flow rate of $1 \mathrm{~mL} / \mathrm{min}$ with UV detector monitoring at $220 \mathrm{~nm}$. The injection volume was $30 \mu \mathrm{L}$ and the column temperature was set at $30^{\circ} \mathrm{C}$. JHIII titers were quantified by comparing the peak areas with those obtained for external quantitative standards ${ }^{24,25}$. Each sample was analyzed three times by HPLC to ensure precision, and the average was used as the JHIII titer for a single sample at a given time point. 


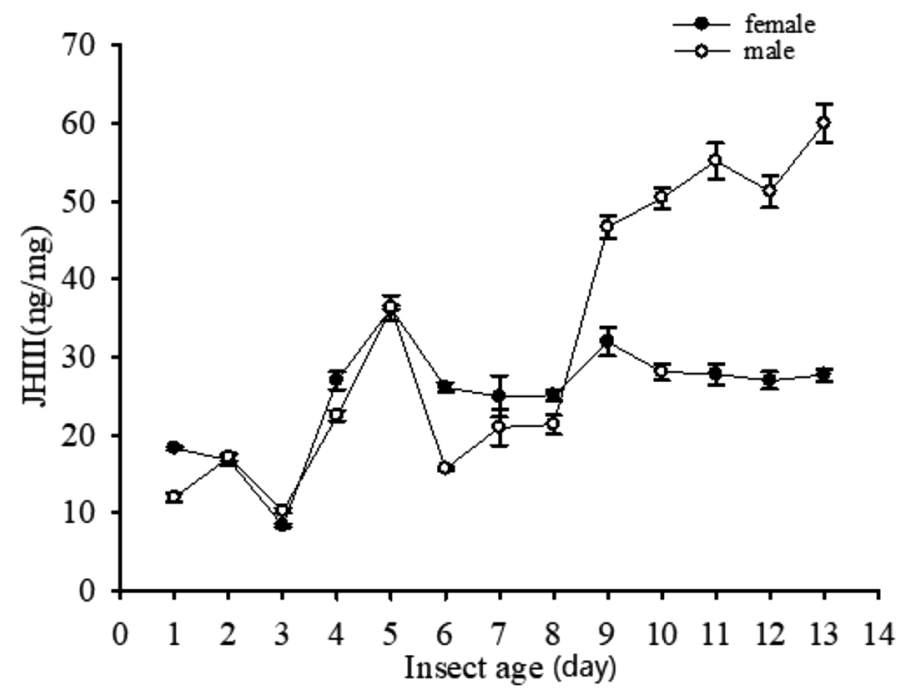

Figure 1. Changes in the juvenile hormone III (JHIII) titer in the absence of ultrasound in Monochamus alternatus adults. Each point represents the mean \pm SE analyzed by HPLC and each HPLC measurement was repeated three times for a single sample to validate the analytical precision. Total number of $M$. alternatus adults across all three replicates: $\mathrm{n}=234$.

We identified and quantified JHIII by reverse phase HPLC. JHIII was identified based on its retention time relative to the JHIII standard but we could not exclude the possibility that other organic compounds might coelute and absorb at the same wavelength as JHIII. Therefore, the JHIII titers might have been lower than those determined in this study ${ }^{25}$.

Data analysis. The decrease in the JHIII titer was calculated as the JHIII titer in the control group minus the JHIII titer in the test group divided by the JHIII titer in the control group and multiplied by $100 \%$. All data were analyzed by one-way analysis of variance using GraphPad Prism (version 4.0 for Mac, GraphPad Software, San Diego, California, USA). The results were expressed as the mean \pm SE. Tukey's multiple comparison test was performed when significance differences were detected at $P<0.05$.

\section{Results}

In this study, we measured the JHIII titers in M. alternatus adults (Fig. 1). In the absence of ultrasound, we found that the JHIII titers in M. alternatus adults differed according to sex. On the day of emergence, the JHIII titer in female adults was only slightly higher than that in males. On days 1-2, the JHIII titers were similar in both sexes. From day 3 to day 5 , the JHIII titers increased sharply in females and males. Female adults had the highest titer after 5 days but the titer then decreased gradually over the next 8 days. The JHIII titer in males decreased on day 6 , but then increased slightly until 13 days.

When the beetles were exposed to ultrasound produced from the LHC20 system for different periods, we found that the decreases in the JHIII titers varied significantly (Fig. 2), but not significantly between males and females. For the 1-day-old female and male adults, highly significant differences $(P<0.01)$ were found in the mean decreases in the JHIII titers after ultrasound treatment for $1 \mathrm{~h}, 12 \mathrm{~h}$, and $24 \mathrm{~h}$. For the 3 -day-old female and male adults, the mean $( \pm \mathrm{SE})$ decreases in the JHIII titers after ultrasonic treatment for $12 \mathrm{~h}$ were $34.61 \% \pm 2.12 \%$ and $37.26 \% \pm 2.78 \%$, respectively, which were significantly higher $(P<0.01)$ than those after exposure times of $1 \mathrm{~h}$ and $24 \mathrm{~h}$. In 5-day-old female and male adults, the mean $( \pm \mathrm{SE})$ decreases in the JHIII titers after ultrasonic treatment for $12 \mathrm{~h}$ were $32.14 \% \pm 3.71 \%$ and $35.08 \% \pm 2.33 \%$, respectively, which were also significantly higher $(P<0.05)$ than those after exposure for $1 \mathrm{~h}$ and $24 \mathrm{~h}$. Thus, the greatest decreases in the JHIII titers in M. alternatus adults occurred after exposure to ultrasound for $12 \mathrm{~h}$.

We also found that the decreases in the JHIII titers varied significantly (Fig. 3) when beetles with different ages were exposed to ultrasound produced using the LHC20 system for the same amount of time. After ultrasonic treatment for $1 \mathrm{~h}$ and $12 \mathrm{~h}$, the mean $( \pm \mathrm{SE})$ decreases in the JHIII titers were significantly higher in the 5 -day-old adults $(P<0.05)$ than the 1 -day-old or 3-day-old adults. However, after ultrasonic treatment for $24 \mathrm{~h}$, the mean $( \pm \mathrm{SE})$ decreases in the JHIII titers were significantly lower in 3 -day-old adults $(P<0.01)$ than 1 -dayold or 5-day-old adults. These results indicated that ultrasonic treatment had different stressful effects on the endocrine regulation system in $M$. alternatus adults depending on their age.

Ultrasound production by both devices significantly reduced the JHIII titers in M. alternatus adults, but there were no significant differences between males and females (Fig. 4). The decreases in the JHIII titers in male beetles were $23.86 \% \pm 1.20 \%, 78.64 \% \pm 7.13 \%$, and $46.25 \% \pm 3.04 \%$, whereas those in female beetles were $26.46 \% \pm 1.84 \%, 68.87 \% \pm 6.16 \%$, and $47.35 \% \pm 7.05 \%$ after exposure to ultrasound generated by the function generator or $\mathrm{LCH} 20$ for $12 \mathrm{~h}$, respectively. Ultrasound at a frequency of $60 \mathrm{kHz}$ resulted in the greatest decrease in the JHIII titers $(P<0.01)$ compared with the other ultrasound frequencies. 

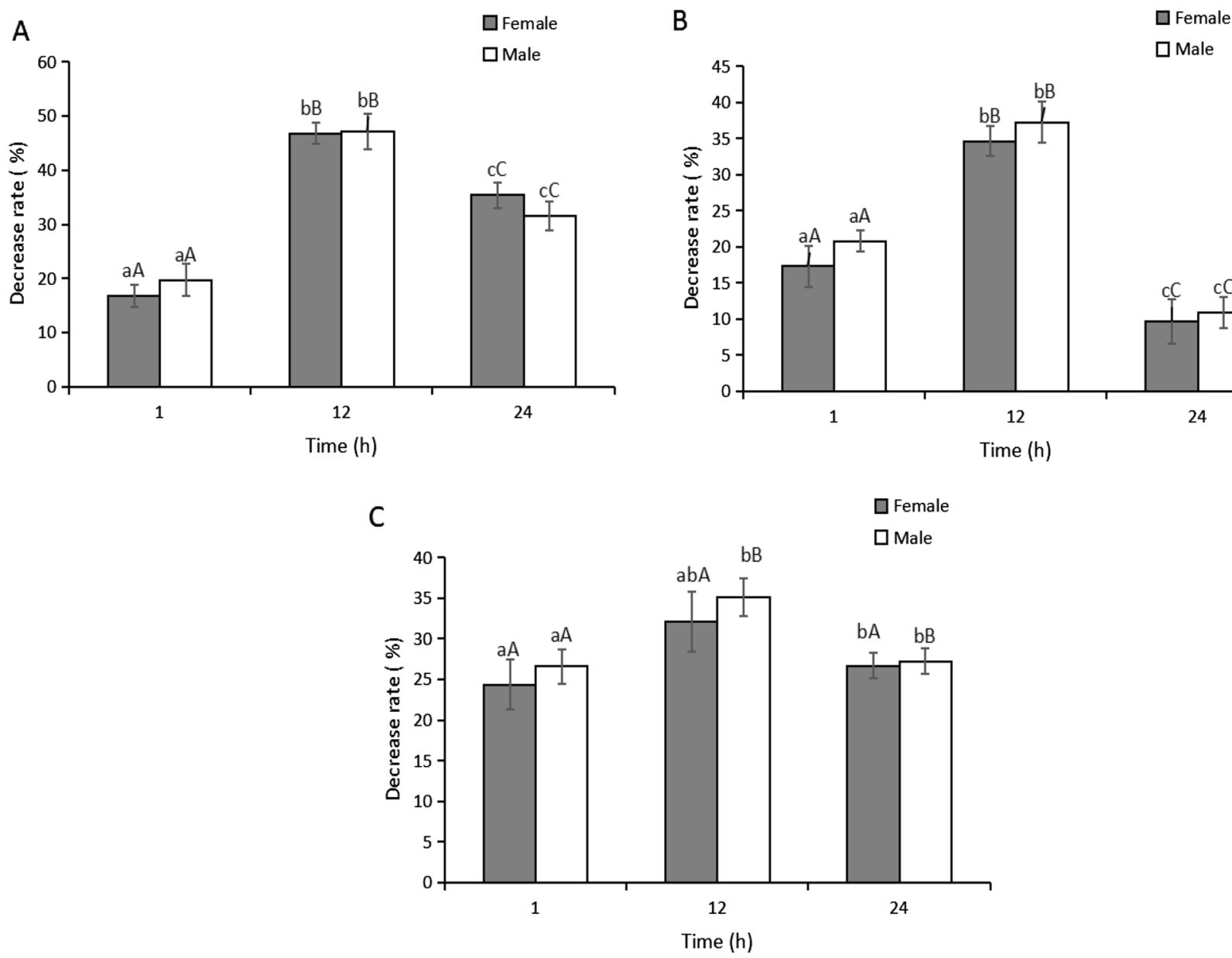

Figure 2. Decrease rate of juvenile hormone III titers of Monochamus alternatus adults during different period of time in the presence of ultrasound of LHC20. Bars are the mean \pm SE for $n=324$ of total numbers of $M$. alternatus adults across all three replicates. Different letters with the different bars indicate significant differences at the 5\% and 1\% level. (A) 1-day-old M. alternatus adults; (B) 3-day-old M. alternatus adults; (C) 5-day-old M. alternatus adults.

\section{Discussion}

$\mathrm{JH}$ plays various roles in developmental and physiological processes in adult insects ${ }^{26-28}$. In particular, changes in the JH titer are essential for reproductive maturation in insects. After emergence, the JH titer increases significantly from the 7 th day in L. migratoria females ${ }^{6}$, whereas it increases gradually from the 11 th day in Schistocerca gregaria (Forskai) (Orthoptera: Acrididae) males $^{29}$, the similar trend was found in Onthophagus taurus

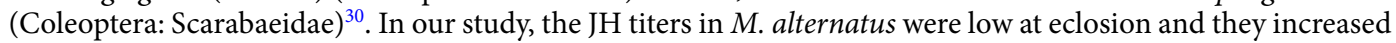
from the third day after adult emergence. Female adults had the highest titers when they were 5 days old, but they then decreased gradually over the next 8 days. The JHIII titers in males decreased on day 6 , but then increased slightly until day 13 (Fig. 1). These results may help to explain why 5-day-old males can copulate successfully and 6-day-old females can oviposit eggs ${ }^{31}$.

Various changes in the JH titers occur in both sexes in M. alternatus adults, and we consider that these changes are necessary for oviposition based previous studies ${ }^{6,731}$. The differences in the JH titers of $M$. alternatus males and females are probably important for the development of the sexes. The rates of maturation and reproductive behavior also differ in M. alternatus males and females. The JH levels increase after emergence and initiate vitellogenesis, mating, and other processes ${ }^{32}$.

The JH titers can vary according to various internal (e.g., hormonal and genetic) and external (e.g., temperature and nutritional) factors $\mathrm{s}^{33,34}$. The photoperiod and temperature may greatly influence the biosynthesis of $\mathrm{JH}$ and sexual maturation in the cotton bollworm ${ }^{35}$.

Sound stress is generally considered to be an environmental stress with severe impacts on animals and humans. Ultrasound produced from the LHC20 device was perceived as a sound stress by M. alternatus. Compared with the control group, we found that the $\mathrm{JH}$ titers decreased significantly under ultrasound treatment (Figs. 2 and 3), and these results are consistent with our previous studies ${ }^{17,22}$. Ultrasound stress can significantly influence the activity of acetylcholinesterase in $H$. armigera ${ }^{17}$. The cholinergic system in M. alternatus adults is also modulated by ultrasound stress ${ }^{22}$. Similar effects of ultrasound stress on insects were observed in other 

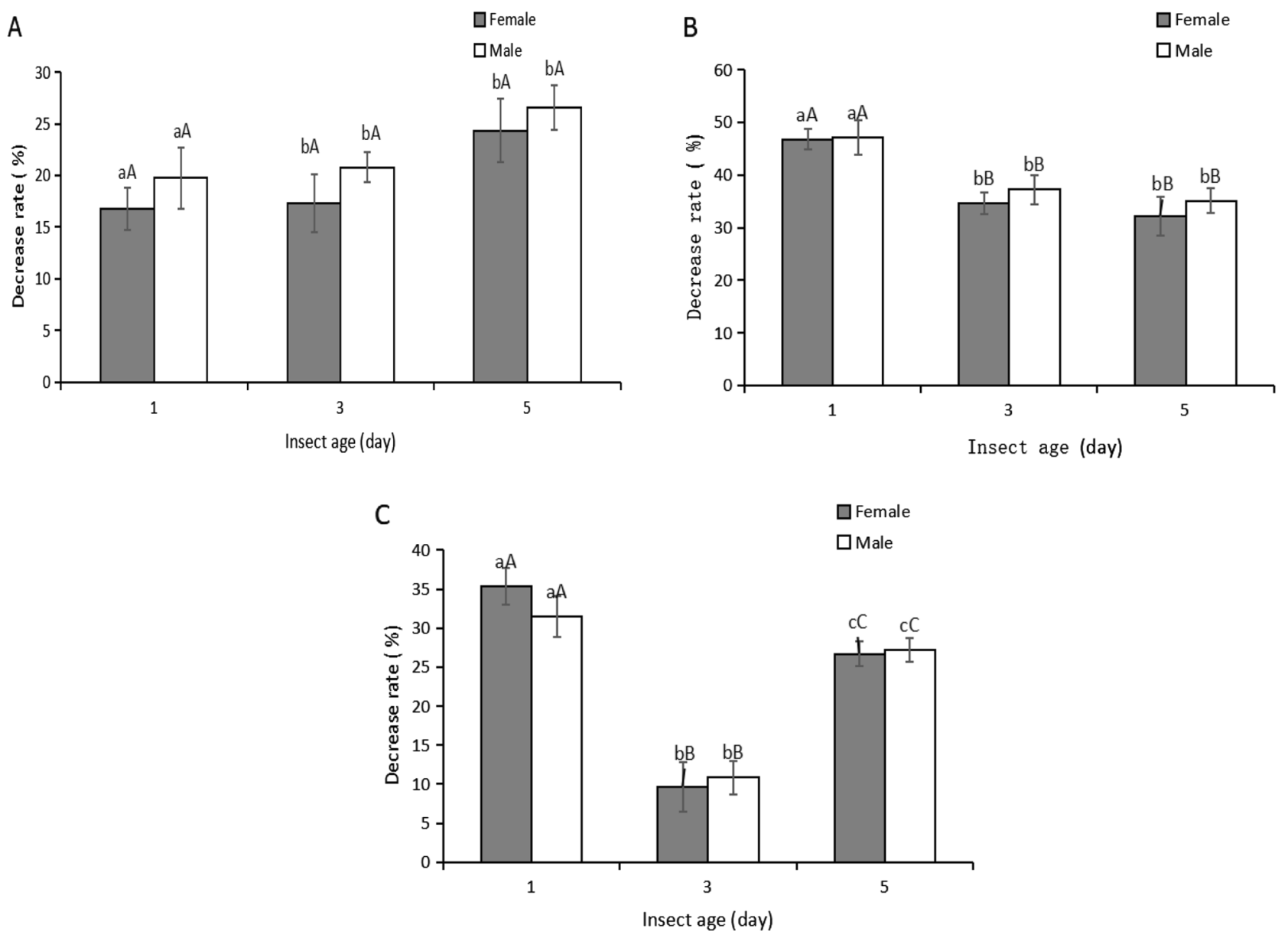

Figure 3. Decrease rate of juvenile hormone III titers of Monochamus alternatus adults with difference ages in the presence of ultrasound of LHC20. Bars are the mean \pm SE for $n=324$ of total numbers of M. alternatus adults across all three replicates. Different letters with the different bars indicate significant differences at the $5 \%$ and $1 \%$ level. (A) $1 \mathrm{~h}$ ultrasound exposed; (B) $12 \mathrm{~h}$ ultrasound exposed; (C) $24 \mathrm{~h}$ ultrasound exposed.

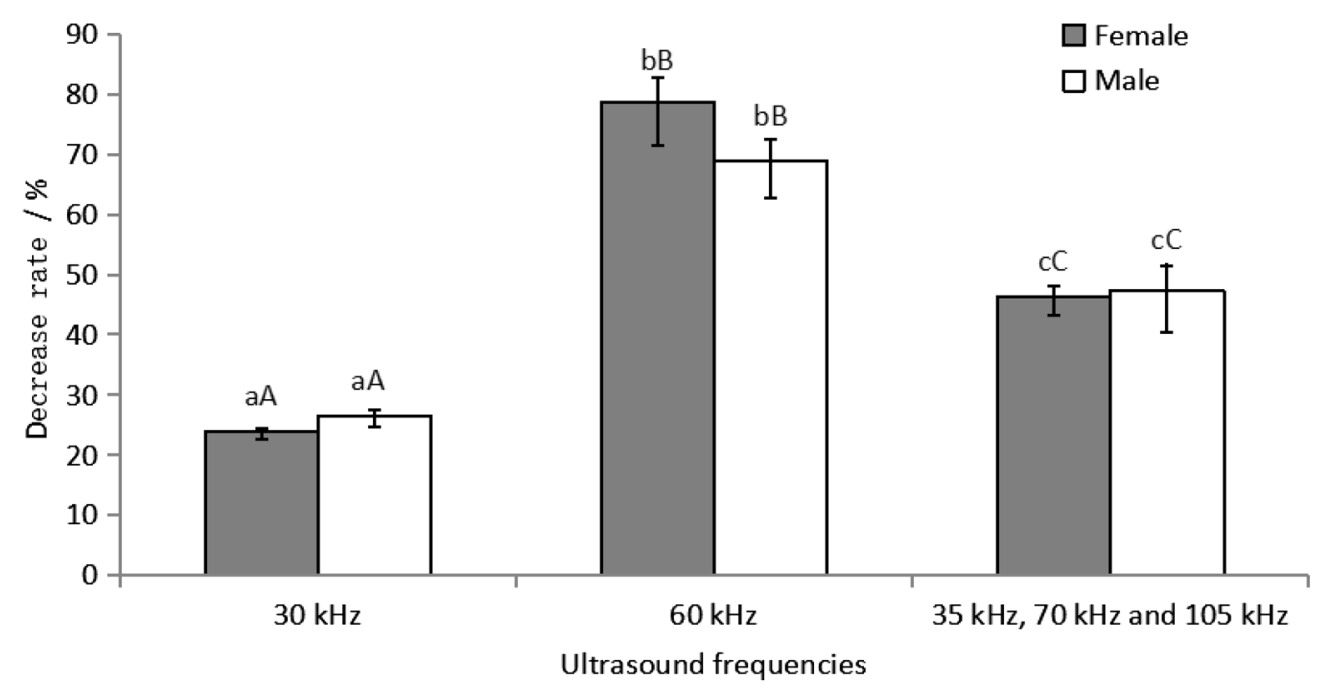

Figure 4. Decreases in juvenile hormone III titers in Monochamus alternatus adults after exposure to ultrasound at different frequencies. Different letters on bars indicate significant differences at $P<0.05$. Data obtained for the same exposure time were analyzed by one-way analysis of variance, and the least significant difference test was used to compare means. Bars represent the mean $\pm \mathrm{SE}$ for $\mathrm{n}=108$ M. alternatus adults across all three replicates. 
studies $^{16,36-38}$. These results suggest that ultrasound can produce an environmental stress effect on $M$. alternatus and act as an external stimulatory factor that might affect the hormone metabolism system in adults.

In the present study, we compared the JHIII titers in M. alternatus adults exposed to ultrasound at different frequencies (Fig. 4). We found that exposure to ultrasound at a frequency of $60 \mathrm{kHz}$ reduced the JHIII titers to the lowest level and this frequency is in the range of predatory bats. Thus, the ultrasound frequencies produced by bats might influence the physiological activities of insects ${ }^{39}$. Our results showed that different ultrasound frequencies, especially bat-like frequencies, could significantly decrease the JHIII titers in M. alternatus adults, thereby disrupting the normal JHIII titers.

Many previous studies of $\mathrm{JH}$ metabolism have focused on $\mathrm{JH}$ esterases ${ }^{40}$. The regulation of $\mathrm{JH}$ production in adult insects is a complex process that involves endogenous neuroendocrine signals ${ }^{41}$. Our results suggest that ultrasound might disrupt the balance of JH synthesis and degradation, but identifying the specific molecular mechanisms involved requires further study ("Supplementary Information").

\section{Conclusion}

In the present study, we demonstrated that exposure to ultrasound significantly decreased the JHIII titers in $M$. alternatus adults. The decreases in the JHIII titers due to exposure to ultrasound did not differ between the sexes, but the effects on beetles with different ages varied significantly depending on the duration of exposure. The decreases in the JHIII titers were highest in male and female beetles after exposure to ultrasound for $12 \mathrm{~h}$. Different ultrasound frequencies had variable effects on the decreases in the JHIII titers in $M$. alternatus adults. Exposing beetles to ultrasound at a frequency of $60 \mathrm{kHz}$ resulted in the greatest decrease in the JHIII titers.

Received: 28 June 2020; Accepted: 28 December 2020

Published online: 14 January 2021

\section{References}

1. Giray, T., Giovanetti, M. \& West-Eberhard, M. J. Juvenile hormone, reproduction, and worker behavior in the neotropical social wasp Polistes canadensis. PNAS 102, 3330-3335 (2005).

2. Hernández-Martinez, S., Mayoral, J. G., Li, Y. \& Noriega, F. G. Role of juvenile hormone and allatotropin on nutrient allocation, ovarian development and survivorship in mosquitoes. J. Insect Physiol. 53, 230-234 (2007).

3. Santos, C. G., Humann, F. C. \& Hartfelder, K. Juvenile hormone signaling in insect oogenesis. Curr. Opin. Insect Sci. 31, 43-48 (2019).

4. Wyatt, G. R. Juvenile hormone in insect reproduction-A paradox?. Eur. J. Entomal. 94, 323-333 (1997).

5. Goodman, W. G. \& Cusson, M. The Juvenile hormones. In Insect Endocrinology (ed. Gilbert, L. I.) 310-365 (Academic Press, Inc., Elsevier, Netherlands, 2012).

6. Rose, U., Ferber, M. \& Hustert, R. Maturation of muscle properties and its hormonal control in an adult insect. J. Exp. Biol. 204, 3531-3545 (2001).

7. Parthasarathy, R., Sun, Z., Bai, H. \& Palli, S. R. Juvenile hormone regulation of vitellogenin synthesis in the red flour beetle, Tribolium castaneum. Insect Biochem. Molec. Biol. 40, 405-414 (2010).

8. Park, Y. I., Shu, S., Ramaswamy, S. B. \& Srinivasan, A. Mating in Heliothis virescens: Transfer of juvenile hormone during copulation by male to female and stimulation of biosynthesis of endogenous juvenile hormone. Arch. Insect Biochem. Physiol. 38, 100-107 (1998).

9. Ignell, R., Couillaud, F. \& Anton, S. Juvenile-hormone-mediated plasticity of aggregation behaviour and olfactory processing in adult desert locusts. J. Exp. Bio. 204, 249-259 (2001).

10. Gujar, H. \& Palli, S. Juvenile hormone regulation of female reproduction in the common bed bug, Cimex lectularius. Sci. Rep. 6, 35546 (2016).

11. Anton, S. \& Gadenne, C. Effect of juvenile hormone on the central nervous processing of sex pheromone in an insect. Proc. Natl. Acad. Sci. USA 96, 5764-5767 (1999).

12. Cherbas, L., Koehler, M. M. \& Cherbas, P. Effects of juvenile hormone on the ecdysone response of Drosophila Kc cells. Dev. Genet. 10, 177-188 (1989).

13. Aikawa, T. et al. Longicorn beetle that vectors pinewood nematode carries many wolbachia genes on an autosome. Proc. Biol. Sci. 276, 3791-3798 (2009).

14. Wang, X. R. et al. Technical standard for controlling pine sawyer (Monochamus alteratus Hope) 1-22 (Apple Academic Press, Inc., China Forestry Publishing House, China, 2009) (in Chinese).

15. Mankin, R. W. Applications of acoustics in insect pest management. CAB Rev. 7, 1-7 (2012).

16. Huang, F. \& Subramanyam, B. Behavioral and reproductive effects of ultrasound on the Indian meal moth, Plodia interpunctella. Entomol. Exp. Appl. 113, 157-164 (2004).

17. Zha, Y. P., Fen, X., Chen, Q. C. \& Lei, C. L. Effect of ultrasound on acetyloinesterase activity of Helicoverpa armigera. Can. Entomol. 140, 563-568 (2008).

18. Zha, Y. P. \& Lei, C. L. Effects of ultrasound-stress on antioxidant enzyme activities of Helicoverpa Armigera (Hübner) (Lepidoptera: Noctuidae). J. Agric. Urban. Entomol. 28, 34-41 (2012).

19. Holderied, M. W., Thomas, L. A. \& Korine, C. Ultrasound avoidance by flying antlions (Myrmeleontidae). J. Exp. Biol. 221, jeb189308 (2018).

20. Nishimura, M. Daily observation on behaviors of japanese pine sawyer adult, Monochamus alternatus hope. Jap. Forest. Soc. J. 55, 100-104 (1973).

21. Robinson, M. \& Stebbings, R. Food of the serotine bat, Eptesicus serotinus-Is faecal analysis a valid qualitative and quantitative technique?. J. Zool. 231, 239-248 (1993).

22. Wu, X., Zha, Y., Chen, J. \& Chen, Q. Effect of ultrasound stress on acetylcholinesterase activity of Monochamus alternatus Hope (Coleoptera: Cerambycidae). J. Huazhong Normal Univ. (Nat. Sci.) 48, 240-244 (2014) (in Chinese).

23. Zhu, X., Liu, Y., Shi, L. M., Ye, G. X. \& Feng, J. Characteristics and analysis of echolocation calls by Eptesicus serotinus andersoni. Sichuan J. Zool. 28, 59-63 (2009).

24. Jiang, X. F., Huang, S. H. \& Luo, L. Z. Juvenile hormone changes associated with dispause induction, maintenance, and termination in the beet webworm, Loxostege sticticalis (Lepidoptera: pyralidae). Arch. Insect. Biochem. Physiol. 3, 134-144 (2011).

25. Zhang, L., Luo, L. Z. \& Jiang, X. F. Starvation influences allatotropin gen expression and juvenile hormone titer in the adult oriental armyworm Mythimna separata. Arch. Insect. Biochem. Physiol. 68, 63-70 (2008). 
26. Khalil, S. M. S., Anspaugh, D. D. \& Roe, R. M. Role of juvenile hormone esterase and epoxide hydrolase in reproduction of the cotton bollworm, Helicoverpa zea. J. Insect. Physiol. 52, 669-678 (2006).

27. Li, Y. P., Zhang, Z., Robinson, G. E. \& Palli, S. R. Identification and characterization of a juvenile hormone response element and its binding proteins. J. Biol. Chem. 282, 37605-37617 (2007).

28. Zhang, L., Cheng, L., Chapman, J. W., Sappington, T. W. \& Jiang, X. Juvenile hormone regulates the shift from migrants to residents in adult oriental armyworm, Mythimna separate. Sci. Rep. 10, 11626 (2020).

29. Tawfik, A. I., Treiblmayr, K., Hassanali, A. \& Osir, E. O. Time-course of haemolymph juvenile hormone titres in solitarius and gregarious adults of Schistocerca gregaria, and their relation to pheromone emission, CA volumetric changes and oocyte growth. J. Insect. Physiol. 46, 1143-1150 (2000).

30. Emlen, D. J. \& Nijhout, H. F. Hormonal control of male horn length dimorphism in Onthophagus taurus (Coleoptera: Scarabaeidae): A second critical period of sensitivity to juvenile hormone. J. Insect Physiol. 47, 1045-1054 (2001).

31. Katsunori, N. M. Vector-host tree relationships and the abiotic environment. In Pine WiltDisease (ed. Zhao, B. G.) 144-161 (Springer, Tokyo, 2008).

32. Engelmann, F. Ecdysteroids, juvenile hormone and vitellogenesis in the cockroach Leucophaea maderae. J. Insect Sci. 2, 1-8 (2002).

33. Nijhout, H. F. \& Wheeler, D. E. Juvenile hormones and the physiological basis of insect polymorphisms. Q. Rev. Biol. 57, 109-133 (1982).

34. Flatt, T., Tu, M. P. \& Tatar, M. Hormonal pleiotropy and the juvenile hormone regulation of Drosophila development and life history. BioEssays 27, 999-1010 (2005).

35. Zhou, X., Coll, M. \& Applebaum, S. W. Effect of temperature and photoperiod on juvenile hormone biosynthesis and sexual maturation in the cotton bollworm, Helicoverpa armigera: Implications for life history traits. Insect Biochem. Mol. Biolm. 30, 863-868 (2000).

36. Sevensson, G. P., Skals, N. \& Löfstedt, C. Disruption of the odour-mediated mating behaviour of Plodia interpunctella using highfrequency sound. Entomol. Exp. Appl. 106, 187-192 (2003).

37. Fullard, J. H., Muma, K. E. \& Sawson, J. W. Quantifying an anti-bat flight response by eared moths. Can. J Zool. 81, 395-399 (2003).

38. Skals, N., Plepys, D. \& Löfstedt, C. Foraging and mate-finding in the silver Y moth, Autographa gamma (Lepidoptera: Noctuidae) under the risk of predation. Oikos 102, 351-357 (2003).

39. Greenfield, M. D. \& Baker, M. Bat Avoidance in non-aerial insects: The silence response of signaling males in an acoustic moth. Ethology 109, 427-442 (2003).

40. Minakuchi, C. \& Riddiford, L. M. Insect juvenile hormone action as a potential target of pest management. J. Pestic. Sci. 31, 77-84 (2006).

41. Teal, P. E. A. Effects of allatotropin and allatostatin on in vitro production of juvenile hormones by the corpora allata of virgin females of the moths of Heliothis virescens and Manduca sexta. Peptides 23, 663-669 (2001).

\section{Acknowledgements}

We are grateful to Lindsay Larson and Dr Wuguo Chen (University of North Carolina at Chapel Hill, North Carolina, United States of America) for helpful comments and suggestions concerning the manuscript. This study was supported by the National Key Research and Development Program of China (Grant No. 2017YFD0600100) and the National Science Foundation for Scientists of China (Grant No. 30901154).

\section{Author contributions}

Y.-P.Z. and X.-L.W. wrote the main manuscript text. Y.-P.Z., X.-L.W. and Z.-Y.Z. prepated figures. All authors reviewed the manuscript.

\section{Competing interests}

The authors declare no competing interests.

\section{Additional information}

Supplementary Information The online version contains supplementary material available at https://doi. org/10.1038/s41598-021-81227-2.

Correspondence and requests for materials should be addressed to Y.-P.Z.

Reprints and permissions information is available at www.nature.com/reprints.

Publisher's note Springer Nature remains neutral with regard to jurisdictional claims in published maps and institutional affiliations.

Open Access This article is licensed under a Creative Commons Attribution 4.0 International License, which permits use, sharing, adaptation, distribution and reproduction in any medium or format, as long as you give appropriate credit to the original author(s) and the source, provide a link to the Creative Commons licence, and indicate if changes were made. The images or other third party material in this article are included in the article's Creative Commons licence, unless indicated otherwise in a credit line to the material. If material is not included in the article's Creative Commons licence and your intended use is not permitted by statutory regulation or exceeds the permitted use, you will need to obtain permission directly from the copyright holder. To view a copy of this licence, visit http://creativecommons.org/licenses/by/4.0/.

(C) The Author(s) 2021 